Burke. - Chapman, Gerald W.: Edmund Burke. The Practical Imagination. Cambridge/ Mass./USA: Harvard University Press 1967 . (XIII, 350 S., x Taf., $8^{\circ}$ ) Leinen $\$ 5.95$

Hobbes. - Goldsmith, M.M.: Hobbes's Science of Politics. London W.C. 1 : Columbia University Press I968. (XV, 274 S. gr. $8^{\circ}$ ) Leinen s $67 / 6$

Johnson, Samuel: Collection of Critical Essays. Ed. by Donald J. Greene. (In: Twentieth Century Views.) Englewood Cliffs/N.J./USA: Prentice-Hall International, Inc.I965. (VI, 185 S. $8^{\circ}$ ) Karton. $\$ 1.95$

- Ders.: The Yale Edition of the Work. Vol. VII/VIII: Johnson on Shakespeare. Ed. by Arthur Sherbo. With an introduction by Bertrand H. Bronson. (Two volumes.) New Haven and London: Yale University Press I968. (XLV, 524 S., 2 Faks.; VII S., S. 525I I00; $x$ Faks., gr. $8^{\circ}$ ) Leinen $225 \mathrm{~s}$ net.

Kant. - Schwartländer, Johannes: Der Mensch ist Person. Kants Lehre vom Menschen. Stuttgart: Kohlhammer 1968. (266 S. gr. 80) Karton. DM 42.-

Kofler, Leo: Der asketische Eros. Industriekultur u. Ideologie. (In d. Reihe: Geist u. Gesellschaft.) Wien: Europa Verlag/Frankfurt: Europäische Verlagsanstalt 1967. (340 S. $\left.8^{\circ}\right)$ Karton. DM 9.80

Pentikäinen, Juha: The Nordic dead-child tradition. Nordic dead-child being. A study in comparative religion. (FF Communications. Vol. LXXXV, I. No 202). Helsinki: Suomalainen Tiedeakatemia / Academia Scientiarum Fennica I968. (388 S., 3 Taf., gr. $\left.8^{\circ}\right)$

Rothacker. - Perpeet, Wilhelm: Erich Rothacker. Philosophie des Geistes aus dem Geist der Deutschen Historischen Schule. (Academica Bonnensia. Bd 3.) Bonn: Bouvier \& Co. / L.. Röhrscheid I 968. (I 23 S. I Taf., $8^{\circ}$ ) Karton. DM I4.80

Wyss, Bernhard: Vom verborgenen griechischen Erbe. Rektoratsrede. (Basler Universitätsteden. 57. Heft.) Basel: Helbing \& Lichtenhahn 1968. (34 S. gt. $8^{\circ}$ ) Katton DM 4.-

\title{
Vorbereitung einer bistorisch-kritischen Ausgabe der Werke von Friedrich Maximilian von Klinger (1752-1831)
}

Besitzer von Handschriften Klingers, Briefen an Klinger und zeitgenössischen Drucken von Werken Klingers werden gebeten, sich an einen der Herausgeber zu wenden: Prof. Dr. Sander L. Gilman, Case Western Reserve University, Cleveland, Ohio 44106 USA / Dr. Ulrich Profitlich, Germanisches Seminar der Freien Universität, I Berlin 33, Boltzmannstr. 3 .

\section{Martin-Buber-Briefe gesucht}

Das Martin-Buber-Archiv der Jüdischen National- und Universitätsbibliothek in Jerusalem und das Martin-Buber-Institut der Universität zu Köln bitten alle Besitzer von Briefen Martin Bubers in den deutschsprachigen Ländern und in der CSSR, dem Kölner Institut (D - 5 Köln, Kerpener Str. 4) Kopien der Briefe zur Ergänzung des Archivs und zur Vorbereitung einer Briefausgabe zu überlassen. 